\title{
Neuromyelitis optica spectrum disorder related tonic spasms responsive to lacosamide
}

\author{
A Baheerathan ${ }^{2}$, WJ Brownlee ${ }^{1,2}$, , F Rugg-Gunn ${ }^{2}$, DT Chard ${ }^{1,2,3}$, SA Trip ${ }^{1,2,3}$ \\ ${ }^{1}$ Queen Square Multiple Sclerosis Centre, UCL Institute of Neurology, London, United \\ Kingdom
}

${ }^{2}$ National Hospital for Neurology and Neurosurgery, London, United Kingdom

${ }^{3}$ National Institute for Health Research (NIHR) University College London Hospitals (UCLH)

Biomedical Research Centre, United Kingdom

\section{Correspondence to:}

Dr Anand Trip

Anand.trip@uclh.nhs.uk

Telephone: 02034567890

Fax: 02076762041

\section{Introduction}

Paroxysmal tonic spasms [PTS] are common in patients with neuromyelitis optica spectrum disorder (NMOSD). ${ }^{12}$ In patients with demyelinating disease, PTS can significantly reduce the quality of life, limit activities of daily living and the rehabilitative process following an acute relapse. ${ }^{3}$

As in patients with multiple sclerosis (MS), paroxysmal tonic spasms in NMOSD usually respond well to treatment with carbamazepine. ${ }^{2}$ However, the optimal treatment in patients where carbamazepine is contraindicated or poorly tolerated is unclear. We describe a patient with NMOSD with severe paroxysmal tonic spasms who did not tolerate carbamazepine but was successfully treated with lacosamide (Vimpat).

\section{Case}

A 44 year old woman with NMOSD developed paroxysmal tonic spasms while recovering from an attack of transverse myelitis in August 2015. The tonic spasms were characterised by flexion of the right wrist and elbow, internal rotation of the shoulder and forced head deviation to the right side. Some of the attacks also involved the right leg. Dozens of episodes were occurring each day triggered by any movement of the right arm or head. She was initially treated for spasticity with escalating doses of baclofen, gabapentin and benzodiazepines without improvement. 
When it became apparent that the attacks were tonic spasms, she was started on carbamazepine with prompt resolution but five weeks later developed a generalised exfoliative erythematous rash. The clinical picture was consistent with Stevens-Johnson syndrome with toxic epidermal necrolysis. Carbamazepine was discontinued but within a week severe, frequent tonic spasms recurred.

Alternative sodium-channel blocking agents such as oxcarbazepine, phenytoin and lamotrigine were felt to be contraindicated because of a shared risk of developing StevensJohnson syndrome. She therefore commenced lacosamide $100 \mathrm{mg}$ daily and within 48 hours the tonic spasms were markedly improved and have subsequently ceased with no recurrence of the rash.

\section{Discussion}

PTS are stereotypical, recurrent, localized muscle spasms (in one or more limbs and/or the trunk), lasting between twenty seconds to three minutes, accompanied by severe pain and dystonia. They can have a significant impact on quality of life and rehabilitative potential thus require prompt diagnosis and treatment. ${ }^{3}$ They frequently occur in patients with longitudinally extensive transverse myelitis secondary to NMOSD.

The pathophysiology of PTS is unclear and has been a matter of debate. In demyelinating disease, it is thought that demyelination renders axons hypersensitive to minor insults and subsequent irritation of these axons facilitates ephaptic transmission between demyelinated axons, resulting in PTS. ${ }^{4}$ It has also been hypothesized that dysfunctional ion channels are involved in facilitating ephaptic transmission which may explain why sodium-channel blockers - such as carbamazepine - have been successfully used to suppress PTS in both MS and NMOSD. ${ }^{5}$

Stevens-Johnson syndrome (SJS) and toxic epidermal necrolysis (TENS) account for the most severe forms of cutaneous adverse drug reaction. The pathophysiology of drug-induced SJS is not well understood, however, scientific observations indicate that CD8+ cytotoxic Tcell mediated responses are integral to its pathogenesis. ${ }^{6}$ SJS is a rare but well described adverse reaction to carbamazepine. ${ }^{7}$ Because of the high rate of cross-reactivity with other commonly prescribed anticonvulsants and the previous failure of response to agents used for spasticity, treatment with lacosamide was tried. Lacosamide is a novel anticonvulsant that enhances the slow inactivation of voltage-gated sodium channels and is approved as adjunctive treatment for refractory focal-onset seizures. Although lacosamide can rarely cause a rash, there is no clear association with Stevens-Johnson syndrome. ${ }^{8}$ The clear improvement in paroxysmal tonic spasms seen in this woman with NMOSD suggests that lacosamide can 
be an alternative treatment option in situations where carbamazepine is contraindicated or poorly tolerated.

\section{References}

1. Kim SM, Go MJ, Sung JJ, et al. Painful tonic spasm in neuromyelitis optica: incidence, diagnostic utility, and clinical characteristics. Arch Neurol 2012;69(8):1026-31.

2. Usmani N, Bedi G, Lam BL, et al. Association between paroxysmal tonic spasms and neuromyelitis optica. Arch Neurol 2012;69(1):121-4.

3. Matthews WB. Tonic seizures in disseminated sclerosis. Brain. 1958;81(2):193- 206.

4. Waubant E, Alize P, Tourbah A, Agid Y. Paroxysmal dystonia (tonic spasm) in multiple sclerosis. Neurology 2001;57:2320-2321

5. Espir ME, Millac P. Treatment of paroxysmal disorders in multiple sclerosis with carbamazepine. J Neurol Neurosurg Psychiatry 1970;33:528-531

6. Pavlos R, Mallal S, Ostrov D et al. T-cell mediated hypersensitivity to drugs. Annu Rev Med 2015;66:439-454

7. Khoo AB, Ali F, Yiu Z, et al. Carbamazepine induced Stevens-Johnson syndrome. BMJ Case Reports 2016; doi:10.1136/bcr-2016-214926

8. European Medicines Agency: Vimpat- summary of product characteristics. Available from: http://www.ema.europa.eu/docs/en_GB/document_library/EPAR__Product_Information/human/000863/WC500050338.pdf 